\title{
Survivin protects fused cancer cells from cell death
}

\author{
Mihyang Do ${ }^{1,2,3}$, In-Hae Kwak ${ }^{1}$, Ju-Hyun Ahn ${ }^{1,2,3}$, In Jeong Lee ${ }^{1}$ E Jae-Ho Lee J $^{1,2,3, *}$ \\ ${ }^{1}$ Department of Biochemistry and Molecular Biology, ${ }^{2}$ Genomic Instability Research Center, Ajou University School of Medicine, \\ ${ }^{3}$ Department of Biomedical Sciences, The Graduate School, Ajou University, Suwon 16499, Korea
}

Tetraploidy, a potential precursor of cancer-associated aneuploidy, is produced either by cell fusion or failure of cytokinesis. In this study, low p53-expressing HeLa cells were used to address the fate of cancer cells after fusion. We found that massive cell death or growth arrest occurred a few days after fusion. Interestingly, cells with larger nuclei preferentially died after fusion, suggesting that a larger deviation of DNA content is a strong inducer of apoptosis. Notably, a fraction of cells escaped cell death. Also, the stability of survivin increased, and its localization changed preferentially to the cytosol in fused cells. Knockdown of survivin decreased the survival of fused cells, more than observed in unfused cells, showing increased dependency of fused cells on survivin. Collectively, after cancer cell fusion, some fused cells avoid the apoptotic crisis partly owing to survivin, and continue to proliferate, a process that contributes to human cancer progression. [BMB Reports 2017; 50(7): 361-366]

\section{INTRODUCTION}

Physiological cell fusion results in terminally differentiated cells, such as syncytiotrophoblasts, myocytes and osteoclasts, whereas unphysiological cell fusion induced by various agents, including viruses and chemicals, produce fused cells with proliferative capacity (1). As a result of subsequent cell divisions, these fused tetraploid cells give rise to daughter cells that exhibit genomic instability, a process similar to the genomic instability that follows cytokinesis failure, which results in the daughter cells to become aneuploid and carcinogenic (2).

Unphysiological cell fusion is considered to be a mechanism by which cancer cells acquire more aggressive phenotypes (3). For example, fusion of cancer cells with macrophages confers, on cancer cells, the capacity to invade

${ }^{*}$ Corresponding author. Tel: +82-31-219-5053; Fax: +82-31-2195059; E-mail: jhlee64@ajou.ac.kr

https://doi.org/10.5483/BMBRep.2017.50.7.185

Received 28 October 2016, Revised 17 November 2016, Accepted 14 February 2017

Keywords: Apoptosis, Cell death, Cell fusion, Proliferation, Survivin and metastasize (4). It is also suggested that fusion of cancer cells with endothelial cells may enable cancer cells to more easily penetrate the endothelial cell layer (5). Importantly, fusion between cancer cells induces genomic instability, which is a driving force for these cells to obtain diverse tumor-progression phenotypes (3).

The tetraploid cells, produced by either cell fusion or cytokinesis failure, undergo either cell cycle-arrest or apoptosis through a process considered to be p53 dependent (6-8). Activation of p53 induces p21-dependent cell-cycle arrest, or increases proapoptotic Bcl-2 family proteins, such as Bax and Puma/BBC3, thus inducing apoptosis in a cell contextdependent manner (9-11). Hence, after cell fusion or cytokinesis failure, cells with increased p53 activity are eliminated (8), whereas cells, where p53 activation is limited, survive and even proliferate, demonstrating an ability to form colonies in soft agar (12). Considering the tendency of cancer cells to inactivate p53, fusion between cancer cells results in a high probability of escaping cell cycle arrest and/or cell death after fusion, while simultaneously allowing acquisition of proliferative potential and genomic instability. Therefore, understanding the fate of cells arising from the fusion of cancer cells having decreased p53 activity, is important to understanding the role of cancer cell fusion in cancer progression. In addition, although factors that determine the fate of fused cells are also important, they are yet to be identified.

In this study, we used HeLa cells, which harbor low levels of p53 owing to enhanced p53 degradation in the presence of the E6 viral oncoprotein, as a model system to address the fate of cancer cells after fusion in the context of decreased influence of p53 (13). Interestingly, massive cell death occurred a few days after fusion, followed by the emergence of proliferating cells. These proliferating cells mainly originated from the fusion of two cells, and appeared to have escaped apoptotic cell death, which had otherwise eliminated cells with a higher DNA content. Furthermore, we found that upregulation and cytosolic localization of survivin was partly responsible for the escape of these proliferating cells from apoptotic crisis.

\section{RESULTS}

Fused cells experience massive cell death and growth arrest Separate populations of geneticin-resistant and hygromycin- 
resistant HeLa cells were stained with the vital fluorescence dye $\mathrm{DiO}$ and Dil, respectively, following which they were subjected to electrofusion. Fused cells and unfused cells were separated and isolated by fluorescence-activated cell sorting (FACS). $\mathrm{DiO}(+) / \mathrm{Dil}(+)$ cells were identified as fused cells, whereas $\mathrm{DiO}(-) / \mathrm{Dil}(+)$ cells corresponded to unfused cells, which were used as control cells that had undergone the electrofusion procedure but were without the resultant cell fusion (Supplementary Fig. 1A). Fused and unfused cells were easily differentiated under a fluorescence microscope (Fig. 1A), and FACS analysis revealed that $\sim 99 \%$ of the FACS-sorted fused cells were $\mathrm{DiO}(+)$ and Dil $(+)$ (Supplementary Fig. 1C), indicating the reliability of the FACS procedure. Further analysis of the fused cells immediately after cell fusion revealed that $69.8 \pm 2.7 \%$ had two nuclei, whereas the remaining $\sim 30 \%$ had more than three nuclei, suggesting fusion of more than three cells (Supplementary Fig. 1B).

To address the fate of fused cells, cell proliferation and death were monitored after the fusion. As shown in Fig. 1B, 4 days after cell fusion, the growth rate of fused cells was significantly lower than that of unfused cells. Thereafter, the proliferation of fused cells increased moderately, whereas that of unfused cells increased rapidly. Trypan blue staining revealed that fused cells underwent massive cell death, peaking at $33.7 \pm 4.0 \%$ cell death on day 4 after fusion (Fig. 1C). The subsequent decrease in the cell death rate at day 6 coincided with a gradual increase in the proliferation of fused cells. These observations suggest that the differences observed in cell proliferation might be partly attributable to differences in cell death.

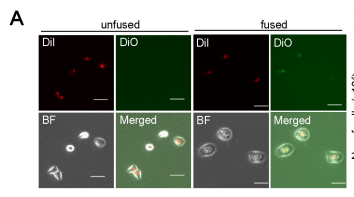

D

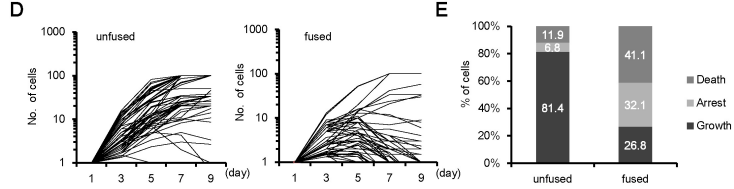

Fig. 1. Analysis of cell fate after cancer cell fusion. (A) Representative images of fused and unfused cells obtained from fusion of $\mathrm{Dil}^{+}-$and $\mathrm{DiO}^{+}$-HeLa cells. HeLa cells were fused as described in Materials and Methods. Bar, $50 \mu \mathrm{m}$. (B) and (C) After cell fusion, cell proliferation and death rate were measured by trypan blue staining. Cells were seeded at a density of $1 \times$ $10^{4}$ cells per well in 12-well plates, and counted at the indicated times. Mean $\pm \mathrm{SD}$ from three independent experiments; $* * * \mathrm{P}<$ $0.001 ; * P<0.05$ by Student's $t$-test. (D) The fate of individual cell was monitored microscopically. (E) At 9 day after cell seeding, cell clones were classified as either growth (wells having more than two cells/well), arrest (1-2 cells/well), or death (no cells in the well, but used to have cells at earlier time points).
We subsequently monitored the fate of individual cells by counting cell numbers over time, in each well of a 96-well plate, after limiting dilution. Whereas most unfused cells showed a steep increase in cell number per well during this period (Fig. 1D, left panel), a majority of fused cells showed a decline in cell number per well after variable times of division (Fig. 1D, right panel). An analysis of clones of unfused cells, 9 days after fusion, classified $81.4 \%$ as proliferative, $6.8 \%$ as growth arrested, and $11.9 \%$ were positioned in the cell death category. The classification of clones of fused cells revealed a different picture: $26.8 \%$ were proliferative, $32.1 \%$ were in growth arrest, and $41.1 \%$ were classified to the cell death category (Fig. 1E). These data clearly demonstrate that despite using cancer cells with decreased p53 activity, a major population of fused cells undergo cell death or growth arrest, whereas a fraction escapes the apoptotic crisis and continues to proliferate.

\section{Elimination of cells with multinucleated nuclei through apoptosis}

To more precisely describe cell fate after cell fusion, we

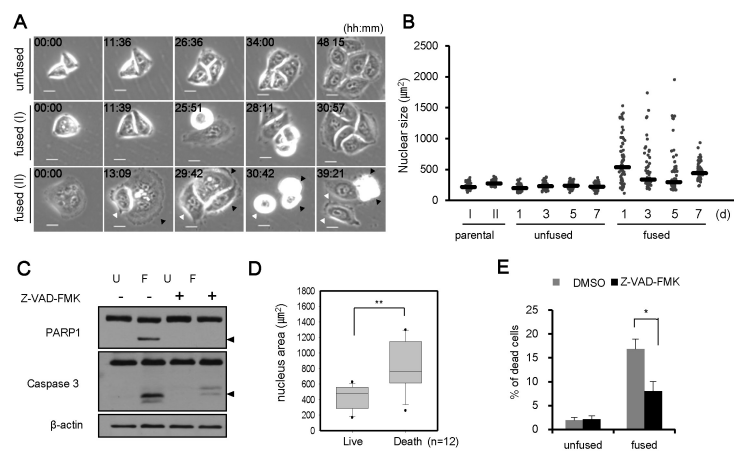

Fig. 2. Preferential elimination of the cells with larger nuclei through apoptosis. (A) Representative images of each cell fate. Time-lapse phase-contrast images captured at the indicated time points show that some daughter cells that originated from fused cells undergo apoptosis (black arrow in row 3), whereas others proliferate like unfused cells (white arrow in row 3). Bar, $20 \mu \mathrm{m}$. (B) Change of nuclear size following cell fusion. Images of DAPIstained nuclei were measured using Axiovision Rel 4.5 software $(\mathrm{n}=700)$. Bar indicates median value. (C) Immunoblots of fused and unfused cells were probed with antibodies to PARP-1 and caspase 3. The level of cleaved PARP-1 and activated caspase 3 (black arrows) increased in the fused HeLa cells. Cells were treated with or without $z-V A D$ fmk $(10 \mu \mathrm{M})$ and harvested at day 3 following cell fusion. ( $\beta$-actin: loading control). (D) Preferential death of cells with larger nuclei. The size of nucleus of cells at interphase was measured by microscopic cell images (Nikon Ti-E) for 4 days following cell fusion. For data analysis, we used the NIS elements software. Supplementary figure 2A shows this in more detail. (E) Cell death was partially abrogated by the $z$-VAD-fmk in fused cells. Cells were seeded in a 12-well plate at a density of $1 \times 10^{4}$ cells/well, and counted by $0.4 \%$ trypan blue staining 3 days after cell fusion. Mean \pm SD from three independent experiments; ${ }^{*} \mathrm{P}<0.05$ by Student's $t$-test. 
continuously monitored the cells by time-lapse microscopy. This analysis clearly revealed a major population of dead (Fig. $2 \mathrm{~A}$, row 3 ) or growth-arrested cells, and a minor population of continuously proliferating cells (Fig. 2A, row 2). In contrast, most unfused cells were proliferative (Fig. 2A, row 1 ). We then addressed whether DNA content affected the fate of fused cells. Since it is difficult to directly measure DNA content from time-lapse data using GFP-H2B- and DsRed-H1-HeLa cells, we instead measured nuclear size as a surrogate marker for DNA content. As expected, the extent of the increase in nuclear size 1 day after fusion varied considerably. Interestingly, the heterogeneity of nuclear size observed on the day after fusion decreased gradually, together with a decrease in the average nuclear size (Fig. 2B), suggesting the disappearance of cells with larger nuclei. Indeed, tracking the fate of daughter cells having same parent cells by time-lapse analysis enabled us to clearly determine that dead cells had larger nuclei compared with live cells (Fig. 2D).

It has previously been reported that tetraploid cells induced by cell fusion or cytokinesis failure tend to die via apoptosis $(14,15)$. To address this aspect, we assessed apoptosis by measuring the levels of the active (cleaved) form of the apoptosis-inducing factors, poly (ADP-ribose) polymerase 1 (PARP-1) and caspase-3, in fused and unfused cells. As shown in Fig. 2C, cleavage of PARP-1 and caspase-3 robustly increased in fused cells but not in unfused cells, suggesting that the death of fused cells was at least partly attributable to apoptosis. Moreover, z VAD-fmk, a pan-caspase inhibitor, clearly abolished the cleavage of both PARP-1 and caspase-3 (Fig. 2C), and partly prevented the death of fused cells, but not unfused cells (Fig. 2E), thereby suggesting a partial involvement of caspase-dependent apoptosis in the death of fused cells. Interestingly, both immunoblot and immunocytochemical assessment of p53 shows an increase in p53 levels in fused cells, as compared to unfused cells (Supplementary Fig. 3), indicating that even in HeLa cells, p53 levels increase after fusion and probably contributed to the massive cell death after cell fusion, thus suggesting that the decrease in p53 in HeLa cells caused by HPV E6 protein can be overcome by strong apoptotic stimuli, one of which is cell fusion.

To confirm the above hypothesis, we evaluated the effect of p53 depletion on growth and death of fused cells. After p53 depletion (Supplementary Fig. 3C), cell growth significantly increased in fused cells after day 5 post-fusion, while there were no changes due to p53 depletion in unfused cells (Supplementary Fig. 3D). In addition, the death of fused cells after p53 depletion decreased at day 3 post-fusion, and significantly dropped at day 5 . On the contrary, unfused cells did not show significant decrease in cell death due to p53 depletion (Supplementary Fig. 3E). Collectively, our data indicate that a majority of fused cells succumbed to death, probably owing to an increase in p53, whereas only a few cells that overcame this apoptotic crisis ultimately attained the capacity to grow continuously.

\section{Survivin is necessary for the survival of fused cells that escape apoptotic crisis}

Since a major fraction of fused cell death was through caspase-dependent apoptosis, averting apoptosis might be very important for the cells that escape this crisis after cell fusion. We therefore measured the expression of various anti- and pro-apoptotic proteins after cell fusion (data not shown). As shown in Fig. 3A, the expression level of survivin, a wellknown anti-apoptotic protein, was clearly increased in fused cells compared to unfused cells, as early as 3 days after fusion, and remained elevated throughout the experimental period. Interestingly, Bcl2 expression increased on day 7 after fusion in both unfused and fused cells, for reasons that are not yet clear. To address whether the increased expression of survivin is a characteristic of surviving fused cells, we measured the survivin expression levels in stable clones of fused cells

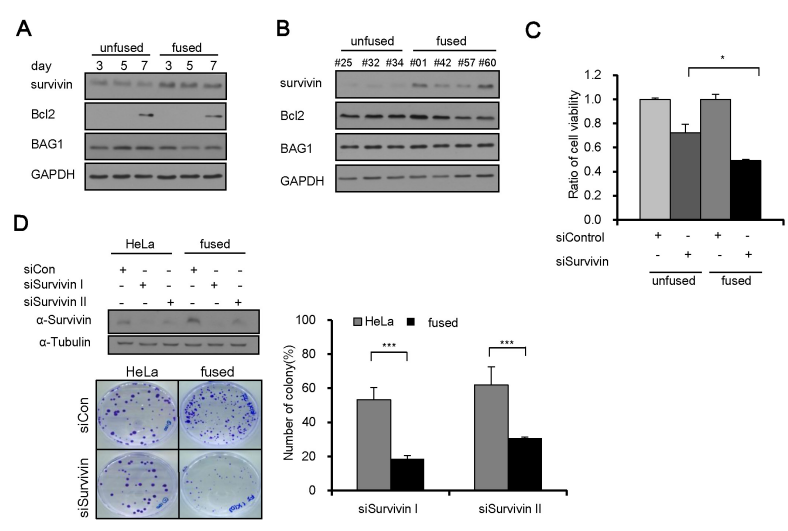

Fig. 3. Survivin increased in surviving fused cells. (A) Cells were harvested at the indicated time points following cell fusion. Protein blots were probed with indicated antibodies. (GAPDH: loading control). (B) Immunoblotting was performed with cell lysates derived from fused or unfused stable lines, which were established by using selective medium containing G418 $(1 \mathrm{mg} / \mathrm{ml})$ and hygromycin $(0.8 \mathrm{mg} / \mathrm{ml})$ for 3 weeks. Numbers indicate different stable clones. (C) Cell viability was quantified by using time-lapse images. Cells were transfected with indicated siRNAs the day after fusion, and monitored using time-lapse microscope for 60 hours. Cell death events were counted and expressed relative to unfused siControl-transfected cells. $n=10$ for each group. Mean $\pm S D$ from triplicate experiments; $* P<0.05$ by Student's t-test. (D) Effect of survivin on the survival of fused cells. Parental HeLa cells, or HeLa cells after electrofusion but without FACS sorting, were transiently transfected with siSurvivin (40 nM) and siControl (40 nM) 1 day after fusion. After $24 \mathrm{~h}$, cells were reseeded and cultured with or without selective medium for 10 days, and colonies were counted after crystal violet staining (left lower panel; a representative picture, right panel; graph showing the relative colony number compared to siControl). At $24 \mathrm{~h}$ after transfection, cell lysates from the indicated samples were subjected to western blot analysis using indicated antibodies (left upper panel). ( $\alpha$-tubulin: loading control). Mean \pm SD from three independent experiments; $* * P<0.001$ by Student's $t$-test. 
previously established by the limiting-dilution procedure. As shown in Fig. 3B, all four established cell lines of fused cells showed a variable, but clear, increase in the expression of survivin, but not $\mathrm{BCl} 2$ or $\mathrm{BAG} 1$, compared with stable clones of unfused cells, strongly suggesting that overexpression of survivin is a common characteristic of surviving fused cells.

Moreover, in colony-forming assay, two different small inhibitory RNA (siRNA) that effectively decreased survivin expression, reduced the survival fraction of fused cells by $70-80 \%$ compared to control siRNA treatment, whereas knockdown of survivin in unfused HeLa cells resulted in approximately a 40-50\% decrease in the survival fraction after fusion compared with control siRNA treatment (Fig. 3D). Therefore, although siSurvivin reduced cell survival in unfused cells, it exerted more effects on the survival of fused cells. Further analysis using time-lapse monitoring confirmed that knockdown of survivin reduced the cell survival significantly more in fused cells, as compared to unfused cells (Fig. 3C). These data suggest that the fused cells that overexpress survivin have the potential to avert the apoptotic crisis of fused cells, and thereby survive to become more stable cells that can proliferate continuously.

\section{Survivin protein in fused cells is localized in the cytosol and shows increased stability}

We subsequently examined how survivin increased in fused cells. The quantitative RT-PCR analysis clearly showed no significant increase in survivin mRNA in cells following fusion (Supplementary Fig. 2B), suggesting a post-transcriptional mechanism. Therefore, we compared survivin degradation rates in fused and unfused cells. In unfused cells, the half-life $\left(t_{1 / 2}\right)$ of survivin protein was less than 30 minutes $(26.3 \pm 3.9$ min), in agreement with previously published reports (16). However, in the case of fused cells, $\mathrm{t}_{1 / 2}$ increased significantly $(48.4 \pm 3.0$ min, Fig. 4 C).

Since cytoplasmic and/or mitochondrial survivin is considered to be cytoprotective (17), we assessed the subcellular localization of survivin by western blotting and immunocytochemical analysis. Interestingly, we observed an increase of survivin in the cytoplasmic fraction of fused cells in western blotting, resulting in approximately a 3 -fold increase in the survivin cytosolic-to-nuclear ratio (Fig. 4A). Immunocytochemical analysis further confirmed the increase of cytosolic survivin in fused cells (Fig. 4B). These data clearly suggest that both the increase in protein stability of survivin and preferential localization to the cytosol, contribute to the survival of some fractions of fused cells.

\section{DISCUSSION}

Tetraploidy is accepted as a potential precursor of cancerassociated aneuploidy, and considered to be a possible cause of tumor formation as well as tumor progression. Tetraploid cells are produced either by cell fusion or cytokinesis failure.
As a first step to understand the implication of cancer cell fusion in tumor progression, we tried to understand the fate of fused cancer cells and the underlying molecular mechanisms related with cell fate. Previous researchers have already shown that most tetraploid cells resulting from non-cancerous cell fusion undergo p53-dependent cell cycle arrest or apoptosis (6). We used HeLa cells as a model system, since HeLa cells are known to have the HPV E6 protein (13), thus possessing low amount/activity of p53, which condition frequently encountered in cancer. Interestingly, we found that even in HeLa cells, massive apoptotic cell death or growth arrest occurred a few days after fusion (Fig. 1 and 2), and surprisingly, it was accompanied by an increase in p53 (Supplementary Fig. 3). Induction of p53 and p53-induced cell death processes in HeLa cells has been reported previously $(18,19)$, suggesting that strong apoptotic stimuli could overcome E6-induced downregulation of p53. In addition, we observed that fused cells with larger nuclei, indicating greater DNA contents, preferentially die after fusion (Fig. 2D), suggesting that a larger deviation from normal DNA content is a strong inducer of
A
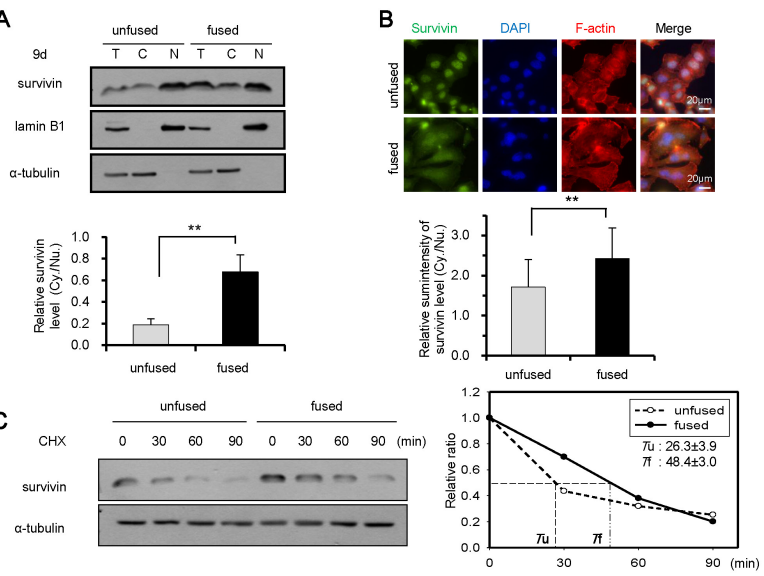

Fig. 4. Increased protein stability and cytosolic localization of survivin in fused cells. Fused and unfused cells were cultured using selective medium containing G418 $(1 \mathrm{mg} / \mathrm{ml})$ and hygromycin $(0.8 \mathrm{mg} / \mathrm{ml})$ for 9 days, and harvested at day 9 following cell fusion. (A) The cytoplasmic and nuclear extracts were subjected to western blotting using survivin, lamin B1 and $\alpha$-tubulin antibodies. (Lamin B1 and $\alpha$-tubulin: fractionation and loading controls). The bar graph shows the quantification of western blot images (Cytoplasmic/Nuclear extracts). Mean \pm SD from three independent experiments; $* * P<0.01$ by Student's $t$-test. (B) Immunofluorescence analysis of fused and unfused cells using survivin and F-actin antibodies. Bar, $20 \mu \mathrm{m}$. Quantification of survivin localization was performed using NIS-Elements Imaging Software (Nikon Corporation). Mean \pm SD from three independent experiments; ${ }^{* * P}<0.01$ by Student's $t$-test. (C) Cells were harvested 5 days after cell fusion, and the stability of survivin protein was analyzed by Western blotting of the whole cell lysates prepared from cells after addition of $50 \mu \mathrm{M}$ cycloheximide (CHX). ( $\alpha$-tubulin: loading control). Relative levels of survivin were measured by densitometric analysis (right panel). 
apoptosis.

Interestingly, a fraction of cells escaped cell death and proliferated, and these surviving fused cells were characterized by upregulation of survivin (Fig. 1, 2 and 3). Survivin is the smallest member of the inhibitor of apoptosis (IAP) family proteins, and plays a key role in inhibiting apoptosis by blocking caspase activation (20). Additionally, survivin is reported to not only exert anti-apoptotic functions, but also cell proliferative functions, reflecting its involvement in forming the chromosome passenger complex, which is crucial for the normal progression of the cell cycle (21). Therefore, the overexpression of survivin probably affects the survival/proliferation of fused cells shortly after fusion, possibly providing the fused cells the ability to overcome the apoptotic crisis.

Regarding the mechanism underlying the increase in survivin, both transcriptional and post-translational regulation were majorly considered (20). It has been reported that the transcription factors SP1, E2F, and HIF-1 $\alpha$ (hypoxia-inducible factor $1 \alpha$ ) increase survivin expression, whereas p53 and the forkhead box transcription factors, FOXO1 and FOXO3, decrease it (20). Notably, hypoxia upregulates both HIF-1 $\alpha$ and survivin expressions in HeLa cells (22). However, our quantitative RT-PCR analysis clearly showed no significant increase in survivin mRNA in cells, following fusion (Supplmentary Fig. 2B). In relation to post-translational regulation, it has been reported that heatshock protein 90 (HSP 90) increases the stability of survivin (23). Although we observed the delay in survivin degradation (Fig. 4), we did not assess the changes in the amount of heatshock protein 90 in fused cells (data not shown). Thus, the molecular mechanism responsible for the upregulation of survivin found here remains undetermined.

Cytoplasmic and/or mitochondrial survivin is considered to be cytoprotective (17). Interestingly, we observed an increase of survivin in the cytoplasmic fraction of fused cells (Fig. 4A and $\mathrm{B}$ ), suggesting that both the increase in protein stability and preferential localization to the cytosol was beneficial in fused cells. However, the inhibition of nuclear export of survivin by using leptomycin B did not decrease the survival of fused cells in our experiments (data not shown), which might be due to the non-specific effects of leptomycin B to the nuclear export of diverse array of proteins other than survivin.

In conclusion, fusion of HeLa cells induces massive apoptosis, despite the fact that the parental cells have low levels of p53. Given that survivin is overexpressed in various malignancies, including breast, lung, prostate, gastric and colon cancers, and high levels of survivin expression correlate with poor prognosis in many cancer patients (24), and the survival of survivin-upregulated cells after cancer cell fusion might contribute to the progression of these types of cancers.

\section{MATERIALS AND METHODS}

See supplementary information.

\section{ACKNOWLEDGEMENTS}

This work was supported by the National Research Foundation of Korea (No. 2010-0011504, 2011-0030043).

\section{CONFLICTS OF INTEREST}

The authors have no conflicting financial interests.

\section{REFERENCES}

1. Duelli DM, Hearn S, Myers MP and Lazebnik Y (2005) A primate virus generates transformed human cells by fusion. J Cell Biol 171, 493-503

2. Ganem NJ, Storchova Z and Pellman D (2007) Tetraploidy, aneuploidy and cancer. Curr Opin Genet Dev 17, 157-162

3. Lu X and Kang Y (2009) Cell fusion as a hidden force in tumor progression. Cancer Res 69, 8536-8539

4. Kerbel RS, Lagarde AE, Dennis JW and Donaghue TP (1983) Spontaneous fusion in vivo between normal host and tumor cells: possible contribution to tumor progression and metastasis studied with a lectin-resistant mutant tumor. Mol Cell Biol 3, 523-538

5. Mortensen K, Lichtenberg J, Thomsen PD and Larsson LI (2004) Spontaneous fusion between cancer cells and endothelial cells. Cell Mol Life Sci 61, 2125-2131

6. Andreassen PR, Lohez OD, Lacroix FB and Margolis RL (2001) Tetraploid state induces p53-dependent arrest of nontransformed mammalian cells in G1. Mol Biol Cell 12, 1315-1328

7. Vogel C, Kienitz A, Hofmann I, Muller R and Bastians $\mathrm{H}$ (2004) Crosstalk of the mitotic spindle assembly checkpoint with p53 to prevent polyploidy. Oncogene 23, 6845-6853

8. Aylon Y and Oren M (2011) p53: guardian of ploidy. Mol Oncol 5, 315-323

9. Vousden $\mathrm{KH}$ and Lu X (2002) Live or let die: the cell's response to p53. Nat Rev Cancer 2, 594-604

10. Yu J, Wang Z, Kinzler KW, Vogelstein B and Zhang L (2003) PUMA mediates the apoptotic response to p53 in colorectal cancer cells. Proc Natl Acad Sci U S A 100, 1931-1936

11. Wei MC, Zong WX, Cheng EH et al (2001) Proapoptotic BAX and BAK: a requisite gateway to mitochondrial dysfunction and death. Science 292, 727-730

12. Ho CC, Hau PM, Marxer M and Poon RY (2010) The requirement of p53 for maintaining chromosomal stability during tetraploidization. Oncotarget 1, 583-595

13. Scheffner M, Werness BA, Huibregtse JM, Levine AJ and Howley PM (1990) The E6 oncoprotein encoded by human papillomavirus types 16 and 18 promotes the degradation of p53. Cell 63, 1129-1136

14. Castedo M, Coquelle A, Vitale I et al (2006) Selective resistance of tetraploid cancer cells against DNA damageinduced apoptosis. Ann NY Acad Sci 1090, 35-49

15. Fukuta $\mathrm{K}$, Kohri $\mathrm{K}$, Fukuda $\mathrm{H}$ et al (2008) Induction of multinucleated cells and apoptosis in the PC-3 prostate 
cancer cell line by low concentrations of polyethylene glycol 1000. Cancer Sci 99, 1055-1062

16. Zhao J, Tenev T, Martins LM, Downward J and Lemoine NR (2000) The ubiquitin-proteasome pathway regulates survivin degradation in a cell cycle-dependent manner. J Cell Sci 113, 4363-4371

17. Li F, Yang J, Ramnath N, Javle MM and Tan D (2005) Nuclear or cytoplasmic expression of survivin: what is the significance? Int J Cancer 114, 509-512

18. Behera B, Mishra D, Roy B et al (2014) Abrus precatorius agglutinin-derived peptides induce ROS-dependent mitochondrial apoptosis through JNK and Akt/P38/P53 pathways in HeLa cells. Chem Biol Interact 222C, 97-105

19. Guo XX, Li Y, Sun C et al (2014) p53-dependent Fas expression is critical for Ginsenoside Rh2 triggered caspase-8 activation in HeLa cells. Protein Cell 5, 224-234
20. Chen X, Duan N, Zhang C and Zhang W (2016) Survivin and Tumorigenesis: Molecular Mechanisms and Therapeutic Strategies. J Cancer 7, 314-323

21. Cheung $\mathrm{CH}$, Huang CC, Tsai FY et al (2013) Survivin biology and potential as a therapeutic target in oncology. Onco Targets Ther 6, 1453-1462

22. Bai H, Ge S, Lu J, Qian G and Xu R (2013) Hypoxia inducible factor-1alpha-mediated activation of survivin in cervical cancer cells. J Obstet Gynaecol Res 39, 555-563

23. Fortugno P, Beltrami E, Plescia J et al (2003) Regulation of survivin function by Hsp90. Proc Natl Acad Sci U S A $100,13791-13796$

24. Altieri DC (2003) Survivin, versatile modulation of cell division and apoptosis in cancer. Oncogene 22, 85818589 